\title{
LINEAR ISOMETRIES BETWEEN SPACES OF FUNCTIONS OF BOUNDED VARIATION
}

\author{
Jesuś Araujo
}

\begin{abstract}
Given two subsets $X$ and $Y$ of $\mathbb{R}$ each with at least two points, we describe the surjective linear isometries between the spaces of functions of bounded variation $B V(X)$ and $B V(Y)$ : namely, if $T: B V(X) \rightarrow B V(Y)$ is such an isometry, then there exist $\alpha \in \mathbb{C}$, $|\alpha|=1$, and a monotonic bijective map $h: Y \rightarrow X$ such that $(T f)(y)=\alpha f(h(y))$ for every $f \in B V(X)$ and every $y \in Y$.
\end{abstract}

Let $X$ be an arbitrary subset of the real line with at least two points. Given a complex valued function $f$ on $X$ we denote by $V(f ; X)$ the variation of $f$ on $X$, that is, the least upper bound of the set

$$
\left\{\sum_{k=1}^{n}\left|f\left(x_{k}\right)-f\left(x_{k-1}\right)\right|: n \in \mathbb{N}, x_{0}, x_{1}, \ldots, x_{n} \in X, x_{0}<x_{1}<\ldots<x_{n}\right\} .
$$

If $V(f ; X)<+\infty$, then $f$ is said to be a function of bounded variation. We denote by $B V(X)$ the set of all functions of bounded variation on $X$. It is straightforward to see that $B V(X)$ becomes a Banach space if we endow it with the norm $\|f\|:=\|f\|_{\infty}+V(f ; X)$, $f \in B V(X)$, where $\|\cdot\|_{\infty}$ stands for the sup norm.

In this paper we give a complete description of the surjective linear isometries between spaces of functions of bounded variation. The techniques used to do this are not based on extreme points or related techniques used to prove similar results in the study of isometries between some other spaces of functions (see for instance [3] or [1]). We use only straightforward concepts, always taking into account that the functions we deal with are not continuous in general. Related results are given for instance in $[2,4]$ and [5], where the authors study the isometries between spaces of absolutely continuous functions, endowed with a similar norm. However, in these papers the fact that the functions are absolutely continuous is fundamental to carrying out their proofs, and no similar approach can be taken in our context.

In the sequel, given a subset $A$ of $\mathbb{C}$, we denote by $\mathrm{cl} A$ its closure in $\mathbb{C}$. Also, for $f \in B V(X)$, we denote by $C(f)$ the set of numbers $\alpha \in \mathbb{C}$ such that $|\alpha|=\|f\|_{\infty}$ and

Received 1st October, 1998

Research supported in part by the Spanish Dirección General de Investigación Científica y Técnica (DGICYT, PB95-0582).

Copyright Clearance Centre, Inc. Serial-fee code: 0004-9729/99 \$A2.00+0.00. 
$\alpha \in \operatorname{cl} f(X)$. For $f \in B V(X), c(f)$ will be the cozero set of $f$, that is, the set of all $x \in X$ such that $f(x) \neq 0$. On the other hand, given an interval $I \subset \mathbb{R}(I \neq \mathbb{R}, \emptyset)$, we say that $I \in \mathfrak{I}_{\leftarrow}$ (respectively $I \in \mathfrak{I}_{\rightarrow}$ ) if it is not bounded below (respectively if it is not bounded above). Finally $T: B V(X) \rightarrow B V(Y)$ will be a surjective linear isometry.

We begin with a straightforward lemma.

LEMma 1. Given $f \in B V(X)$, there exists $\alpha \in \mathbb{C},|\alpha|=1$, such that $\|\alpha f+1\|=$ $\|f\|+1$.

LEMMA 2. T1 is a constant function.

Proof: Suppose that $f \in B V(Y)$. By Lemma 1, taking into account that $T$ is an isometry, we have that there exists $\alpha \in \mathbb{C},|\alpha|=1$, such that $\|\alpha f+T 1\|=\|f\|+1$. With this we have

$$
\begin{aligned}
\|f\|+1 & =\|\alpha f+T 1\|_{\infty}+V(\alpha f+T 1 ; Y) \\
& \leqslant\|f\|_{\infty}+V(f ; Y)+\|T 1\|_{\infty}+V(T 1 ; Y) \\
& =\|f\|+1 .
\end{aligned}
$$

As a consequence we deduce that $\|\alpha f+T 1\|_{\infty}=\|f\|_{\infty}+\|T 1\|_{\infty}$ and

$$
V(\alpha f+T 1 ; Y)=V(f ; Y)+V(T 1 ; Y) \text {. }
$$

In particular this implies that for $y_{0} \in Y$ such that $\left(-\infty, y_{0}\right]$ and $\left[y_{0},+\infty\right)$ have at least two points, we have

$$
V\left(\alpha f+T 1 ; Y \cap\left(-\infty, y_{0}\right]\right)=V\left(f ; Y \cap\left(-\infty, y_{0}\right]\right)+V\left(T 1 ; Y \cap\left(-\infty, y_{0}\right]\right),
$$

and

$$
V\left(\alpha f+T 1 ; Y \cap\left[y_{0},+\infty\right)\right)=V\left(f ; Y \cap\left[y_{0},+\infty\right)\right)+V\left(T 1 ; Y \cap\left[y_{0},+\infty\right)\right)
$$

Next we fix $y_{0}$ as above and define the functions

$$
g_{y_{0}}^{1}:=\xi_{Y \cap\left(-\infty, y_{0}\right]} T 1+(T 1)\left(y_{0}\right) \xi_{Y \cap\left(y_{0},+\infty\right)}
$$

and

$$
g_{y_{0}}^{2}:=\xi_{Y \cap\left(y_{0},+\infty\right)} T 1-(T 1)\left(y_{0}\right) \xi_{Y \cap\left(y_{0},+\infty\right)} .
$$

Let $f_{y_{0}}:=g_{y_{0}}^{1}-g_{y_{0}}^{2}$. We are going to apply the above results. Taking into account that $f_{y_{0}} \equiv T 1$ on $Y \cap\left(-\infty, y_{0}\right]$, we have

$$
V\left((\alpha+1) T 1 ; Y \cap\left(-\infty, y_{0}\right]\right)=2 V\left(T 1 ; Y \cap\left(-\infty, y_{0}\right]\right)
$$

which clearly implies

$$
\alpha=1
$$


whenever $V\left(T 1 ; Y \cap\left(-\infty, y_{0}\right]\right) \neq 0$. As for the other equality, it is clear that $V\left(\alpha f_{y_{0}}+T 1 ; Y \cap\left[y_{0},+\infty\right)\right)$ is equal to

$$
V\left(2 \alpha(T 1)\left(y_{0}\right) \xi_{Y \cap\left(y_{0},+\infty\right)}-\alpha \xi_{Y \cap\left[y_{0},+\infty\right)} T 1+T 1 ; Y \cap\left[y_{0},+\infty\right)\right),
$$

that is,

$$
V\left(\alpha f_{y_{0}}+T 1 ; Y \cap\left[y_{0},+\infty\right)\right)=V\left((1-\alpha) T 1 ; Y \cap\left(y_{0},+\infty\right)\right),
$$

which implies

$$
V\left((1-\alpha) T 1 ; Y \cap\left[y_{0},+\infty\right)\right)=2 V\left(T 1 ; Y \cap\left[y_{0},+\infty\right)\right) .
$$

As a consequence we deduce that $\alpha=-1$ whenever $V\left(T 1 ; Y \cap\left[y_{0},+\infty\right)\right) \neq 0$. Since this is impossible, we conclude that either $V\left(T 1 ; Y \cap\left(-\infty, y_{0}\right]\right)=0$ or $V\left(T 1 ; Y \cap\left[y_{0},+\infty\right)\right)=0$, which means that there exist $I \in \mathfrak{I}_{\leftarrow}$ and $\alpha_{0}, \beta_{0} \in \mathbb{C}$ such that $T 1=\alpha_{0} \xi_{Y \cap I}+\beta_{0} \xi_{Y \cap(\mathbb{R}-I)}$. Note that the same result also holds if $Y$ has just two points.

On the other hand, we can prove that $\left|\alpha_{0}\right|=\left|\beta_{0}\right|$. Otherwise, we suppose for instance that $\left|\beta_{0}\right|<\left|\alpha_{0}\right|$, and take $\gamma_{0} \in \mathbb{C}, \gamma_{0} \neq 0$, such that $\left|\beta_{0}\right|+\left|\gamma_{0}\right|<\left|\alpha_{0}\right|$. If we define $f_{0}:=\gamma_{0} \xi_{Y \cap(\mathbb{R}-I)}$, as we have seen above, there exists $\alpha \in \mathbb{C},|\alpha|=1$ such that $\left\|\alpha f_{0}+T 1\right\|_{\infty}=\left\|f_{0}\right\|_{\infty}+\|T 1\|_{\infty}$. This implies that $\left|\alpha_{0}\right|=\left|\gamma_{0}\right|+\left|\alpha_{0}\right|$, which is not possible. A similar contradiction comes if we assume $\left|\alpha_{0}\right|<\left|\beta_{0}\right|$. Consequently $\left|\alpha_{0}\right|=\left|\beta_{0}\right|$.

Next, note first that we can assume without loss of generality that $\alpha_{0}$ may be taken to be real and positive. We show that if $\alpha_{0} \neq \beta_{0}$, then $\alpha_{0}=-\beta_{0}$. For, take $f_{1}:=$ $\alpha_{0} \xi_{Y \cap I}-\beta_{0} \xi_{Y \cap(\mathbb{R}-I)}$. It is clear that if $\alpha \in \mathbb{C}$ satisfies $\left\|\alpha f_{1}+T 1\right\|_{\infty}=\left\|f_{1}\right\|_{\infty}+\|T 1\|_{\infty}$, then $\alpha=1$ or $\alpha=-1$. We assume that $\alpha=1$, a similar argument being valid also for $\alpha=-1$. Consequently we have $V\left(f_{1}+T 1 ; Y\right)=V\left(f_{1} ; Y\right)+V(T 1 ; Y)$, which means $\left|2 \alpha_{0}\right|=\left|\alpha_{0}+\beta_{0}\right|+\left|\alpha_{0}-\beta_{0}\right|$. This clearly means that $\beta_{0}$ is a real number, since $\alpha_{0}$ is. Also $\left|\beta_{0}\right|=\left|\alpha_{0}\right|$, which implies that $\alpha_{0}=-\beta_{0}$. Finally taking into account that $1=\|T 1\|=\left|\alpha_{0}\right|+\left|2 \alpha_{0}\right|$ we obtain that $\alpha_{0}=1 / 3$.

Now take $f_{2}:=(1 / 3) \xi_{Y \cap I}+(1 / 3) i \xi_{Y \cap(\mathbf{R}-I)}$. We have that there exists $\alpha \in \mathbb{C},|\alpha|=1$, such that $\left\|\alpha f_{2}+T 1\right\|_{\infty}=\left\|f_{2}\right\|_{\infty}+\|T 1\|_{\infty}=2 / 3$. This implies that $\alpha=1$ or $\alpha=i$. Suppose first that $\alpha=1$. For this $\alpha$, we should also have $V\left(\alpha f_{2}+T 1 ; Y\right)=V\left(f_{2} ; Y\right)+$ $V(T 1 ; Y)$, which implies that, making the necessary calculations, $\sqrt{10} / 3=\sqrt{2} / 3+2 / 3$, and this is absurd. A similar argument proves that $\alpha$ cannot be equal to $i$.

We arrive at the conclusion that $\alpha_{0}=\beta_{0}$, this is, $T 1$ is a constant function.

According to Lemma 2, we are going to assume without loss of generality that $T 1=1$ in the rest of the paper.

Lemma 3. Suppose that $\alpha \in C(f), f \in B V(X)$. Then there exists $r>0$ such that $r \alpha \in C(T f)$.

Proof: Without loss of generality, we assume that $\alpha=1$. It is easy to see that $\|f+1\|=\|f\|+1$, and consequently $\|T f+1\|=\|T f\|+1$. Since $V(T f+1 ; Y)=$ 
$V(T f ; Y)$, we have that $\|T f+1\|_{\infty}=\|T f\|_{\infty}+1$. It is easy to see that this implies that there exists $r>0$ such that $r \in C(T f)$.

LEMma 4. Suppose that $\xi_{A} \in B V(X)$ for some $A \subset X$. Then $\left\|T \xi_{A}\right\|_{\infty} \geqslant 1$.

Proof: By Lemma 3, there exists $r>0$ such that $r \in C\left(T \xi_{A}\right)$. Take $f_{0}:=\xi_{A}+\sqrt{3} i$, which clearly satisfies

$$
\left\|f_{0}\right\|-\left\|\xi_{A}\right\|=\left(2+V\left(f_{0} ; X\right)\right)-\left(1+V\left(\xi_{A} ; X\right)\right)=1 .
$$

As $T \sqrt{3} i=\sqrt{3} i$ and $T$ is an isometry, we have that

$$
\begin{aligned}
1 & =\left\|T f_{0}\right\|-\left\|T \xi_{A}\right\| \\
& =\left\|T f_{0}\right\|_{\infty}-\left\|T \xi_{A}\right\|_{\infty} \\
& \geqslant \sqrt{r^{2}+3}-r .
\end{aligned}
$$

Clearly if $r<1$, then $(r+1)^{2}<r^{2}+3$, this is, $1<\sqrt{r^{2}+3}-r$, and the above inequality does not hold. Consequently we deduce that $r \geqslant 1$.

Lemma 5. Let $I$ be an interval. Then there exists an interval $J$ such that $T \xi_{X \cap I}=$ $\xi_{Y \cap J}$. Also if $I \in \mathfrak{I}_{\leftarrow} \cup \mathfrak{I}_{\rightarrow}$, then $J \in \mathfrak{I}_{\leftarrow} \cup \mathfrak{I}_{\rightarrow}$.

PROOF: We prove some claims leading to the result.

Claim 1. Let $I \in \mathfrak{I}_{\leftarrow} \cup \mathfrak{I}_{\rightarrow}$ be such that $X \cap I \neq X, \emptyset$. Then $1 \in C\left(T \xi_{X \cap I}\right)$ and $0 \in \operatorname{cl}\left(T \xi_{X \cap I}\right)(Y)$. Also, if $\alpha \in\left(T \xi_{X \cap I}\right)(Y)$, then $\alpha \in \mathbb{R}$ and $0 \leqslant \alpha \leqslant 1$.

According to Lemmas 3 and $4, C\left(T \xi_{X \cap I}\right)$ contains a real number $r, r \geqslant 1$. Also $f_{0}:=\xi_{X \cap I}-1 / 2$ satisfies $1 / 2,-1 / 2 \in C\left(f_{0}\right)$, and by Lemma 3 , there exist $s_{1}, s_{2} \in C\left(T f_{0}\right)$ with $s_{1}>0$ and $s_{2}<0$. Since $T 1=1$, we have that $s_{1}:=r-1 / 2$. Also it is clear that there exists $r^{\prime} \in \operatorname{cl}\left(T \xi_{X \cap I}\right)(Y)$ such that $s_{2}=r^{\prime}-1 / 2$. It is clear that $r^{\prime} \in \mathbb{R}$, and taking into account that $r \geqslant 1$ and that $r-1 / 2=\left|r^{\prime}-1 / 2\right|$, we deduce that $r^{\prime} \leqslant 0$. On the other hand, since $T$ is an isometry, we have that $\left\|\xi_{X \cap I}\right\|=2=\left\|T \xi_{X \cap I}\right\| \geqslant r+\left(r-r^{\prime}\right)$. This implies clearly that $r=1$ and $r^{\prime}=0$.

Finally suppose that $\alpha$ belongs to $\left(T \xi_{X \cap I}\right)(Y)$. If $\alpha \notin[0,1]$, then $V\left(T \xi_{X \cap I} ; Y\right) \geqslant$ $|\alpha|+|\alpha-1|>1$, which implies that $\left\|T \xi_{X \cap I}\right\|>2$. Since $T$ is an isometry, this is not possible, and we conclude that $\alpha$ is a real number between 0 and 1 .

Claim 2. Let $I$ be an interval. Then $1 \in C\left(T \xi_{X \cap I}\right)$. Also if $\alpha \in\left(T \xi_{X \cap I}\right)(Y)$, then $\alpha \in[-1,1]$.

The result is given by Claim 1 if $I \in \mathfrak{I}_{\leftarrow} \cup \mathfrak{I}_{\rightarrow}$. Otherwise we take $J \in \mathfrak{I}_{\leftarrow}$ such that $J \cap I=\emptyset$ and $J \cup I \in \mathfrak{I}_{\leftarrow}$. It is apparent that $\xi_{X \cap I}=\xi_{X \cap(J \cup I)}-\xi_{X \cap J}$. Also, by Lemmas 3 and 4 , there exists $r \geqslant 1$ contained in $C\left(T \xi_{X \cap I}\right)$. This implies that there exist $r_{1}$ and $r_{2}$ in $\mathrm{cl}\left(T \xi_{X \cap(J \cup I)}\right)(Y)$ and $\operatorname{cl}\left(T \xi_{X \cap J}\right)(Y)$, respectively, such that $r=r_{1}-r_{2}$. Applying Claim 1, we have that $r_{1}, r_{2} \in[0,1]$, which means that $r=1$. 
The proof of the other part of the claim is easy.

Claim 3. Let $I \in \mathfrak{I}_{\leftarrow} \cup \mathfrak{I}_{\rightarrow}$. Then $\left(T \xi_{X \cap I}\right)(Y) \subset\{0,1\}$.

Take $\alpha \in\left(T \xi_{X \cap I}\right)(Y)$. By Claim 1, we know that $\alpha \in \mathbb{R}$ and $0 \leqslant \alpha \leqslant 1$. Now suppose that $0<\alpha<1$. We deduce that there exists $y_{1} \in Y$ such that $\alpha:=\left(T \xi_{X \cap I}\right)\left(y_{1}\right) \neq 0,1$. Now it is clear that $\{\alpha+i\}=C\left(i \xi_{\left\{y_{1}\right\}}+T \xi_{X \cap I}\right)$, and by Lemma 3 there exists $r>0$ such that $r(\alpha+i)$ belongs to $C\left(i T^{-1} \xi_{\left\{y_{1}\right\}}+\xi_{X \cap I}\right)$. It is clear that $r(\alpha+i)$ can be written as $\gamma_{1}+\gamma_{2}$, where $\gamma_{1}$ and $\gamma_{2}$ belong to $\mathrm{cl}\left(i T^{-1} \xi_{\left\{y_{1}\right\}}\right)(X)$ and $\operatorname{cl} \xi_{X \cap I}(X)$, respectively. Now $\gamma_{2} \in\{0,1\}$, and on the other hand we have that $\gamma_{1}=\gamma i$, where $\gamma \in \mathbb{R},|\gamma| \leqslant 1$, by Claim 2. We show that this is impossible. First, if $\gamma_{2}=0$, then $r(\alpha+i)=\gamma i$ is an imaginary number, which is clearly not true. Then we deduce that $\gamma_{2}$ must be equai to $i$. But aiso in inis case we have thai $r(\alpha+i)=i+\gamma i$, which implies $r>1$, since $\alpha \in(0,1)$. On the other hand, the above equality also gives that $r i=\gamma i$, this is, $\gamma>1$. This contradiction yields that $\operatorname{cl}\left(T \xi_{X \cap I}\right)(Y) \subset\{0,1\}$, and the claim is proved.

Now suppose that $I \in \mathfrak{I}_{\leftarrow} \cup \mathfrak{I}_{\rightarrow}$. By Claim 3 we deduce that there exists a subset $J$ of $\mathbb{R}$ such that $T \xi_{X \cap I}=\xi_{Y \cap J}$. Now show that $J$ can be taken to be an interval. For this, it is enough to prove that if $y_{1}, y_{2} \in Y \cap J$ satisfy $y_{1}<y_{2}$, then any $y \in Y$ such that $y_{1}<y<y_{2}$ belongs to $J$. We note that if $y \notin J,\left\|\xi_{Y \cap J}\right\| \geqslant 3$, which is impossible because $\left\|\xi_{Y \cap J}\right\|=\left\|\xi_{X \cap I}\right\|=2$. For a similar reason we deduce that $J$ can be taken in $\mathfrak{I}_{\leftarrow} \cup \mathfrak{I}_{\rightarrow}$

As for the case when $I$ is an interval not in $\mathfrak{I}_{\leftarrow} \cup \mathfrak{J}_{\rightarrow}$, we can work as in the proof of Claim 2. There exists $K \in \mathfrak{I}_{\leftarrow}$ such that $K \cap I=\emptyset$ and $K \cup I \in \mathfrak{I}_{\leftarrow}$. Clearly we have that $\xi_{X \cap I}=\xi_{X \cap(K \cup I)}-\xi_{X \cap K}$. This means that there exist intervals $L$ and $L^{\prime}$ in $\mathfrak{I}_{\leftarrow} \cup \mathfrak{I}_{\rightarrow}$ with $T \xi_{X \cap(K \cup I)}=\xi_{Y \cap L}$ and $T \xi_{X \cap K}=\xi_{Y \cap L^{\prime}}$. Then we have that $T \xi_{X \cap I}=\xi_{Y \cap L}-\xi_{Y \cap L^{\prime}}$. Also by Claim $2,1 \in C\left(T \xi_{X \cap I}\right)$. On the other hand, by Lemma $3,-1 \notin C\left(T \xi_{X \cap I}\right)$. This means that $T \xi_{X \cap I}(Y)=\{0,1\}$, and as a consequence $L^{\prime} \subset L$, this is, $T \xi_{X \cap I}=\xi_{Y \cap\left(L-L^{\prime}\right)}$.

LEMMA 6. If both $I, J$ belong to $\mathfrak{I}_{\leftarrow}$ (respectively to $\left.\mathfrak{I}_{\rightarrow}\right)$, and satisfy $I \subset J$, then $c\left(T \xi_{X \cap I}\right) \subset c\left(T \xi_{X \cap J}\right)$.

Proof: We shall proceed just in the case when $I, J \in \mathfrak{I}_{\leftarrow}$, the other one being similar. The result is trivial if $I=J$, so we suppose $I \neq J$. By Lemma 5 we have that there exist $K, L$ in $\mathfrak{I}_{\leftarrow} \cup \mathfrak{I}_{\rightarrow}$ such that $T \xi_{X \cap I}=\xi_{Y \cap K}$ and $T \xi_{X \cap J}=\xi_{Y \cap L}$. Assuming that $K$ is not contained in $L$, and working as in the end of the proof of Lemma 5 , we arrive at a contradiction. Accordingly $K \subset L$, and we are done.

LEMMA 7. If $I, J$ are intervals satisfying $I \subset J$, then $c\left(T \xi_{X \cap I}\right) \subset c\left(T \xi_{X \cap J}\right)$.

Proof: We shall assume that $I, J \notin \mathfrak{I}_{\leftarrow} \cup \mathfrak{I}_{\rightarrow}$. Take $K, L \in \mathfrak{I}_{\leftarrow}$ such that $I \cap K=$ $\emptyset=J \cap L$ and $I \cup K, J \cup L$ belong to $\mathfrak{I}_{\leftarrow}$. It is clear that $I \cup K \subset J \cup L$, and by Lemma 6 
$c\left(T \xi_{X \cap(I \cup K)}\right) \subset c\left(T \xi_{X \cap(J \cup L)}\right)$. Now we suppose that $y_{0} \in c\left(T \xi_{X \cap I}\right)$. Then we have that

$$
1=\left(T \xi_{X \cap I}\right)\left(y_{0}\right)=\left(T \xi_{X \cap(I \cup K)}\right)\left(y_{0}\right)-\left(T \xi_{X \cap K}\right)\left(y_{0}\right),
$$

which clearly implies that $\left(T \xi_{X \cap(I \cup K)}\right)\left(y_{0}\right)=1$ and $\left(T \xi_{X \cap K}\right)\left(y_{0}\right)=0$, by Lemma 5 . On the other hand, it is easy to see that since $I \subset J$, then $L \subset K$ and $I \cup K \subset J \cup L$. This implies, by Lemma 6 , that $\left(T \xi_{X \cap(J \cup L)}\right)\left(y_{0}\right)=1$ and $\left(T \xi_{X \cap L}\right)\left(y_{0}\right)=0$, that is, $\left(T \xi_{X \cap J}\right)\left(y_{0}\right)=1$. We conclude that $c\left(T \xi_{X \cap I}\right) \subset c\left(T \xi_{X \cap J}\right)$.

The proof of the other cases is similar.

Corollary 8. Let $x_{0} \in X$. Then there exists $y_{0} \in Y$ such that $T \xi_{\left\{x_{0}\right\}}=\xi_{\left\{y_{0}\right\}}$.

Proof: By Lemma 5, we have that there exists an interval $J$ such that $T \xi_{\left\{x_{0}\right\}}=$ $\xi_{Y \cap J}$. Now take $y_{0} \in Y \cap J$. It is clear that by Lemma 7 applied to $T^{-1}, c\left(T^{-1} \xi_{\left\{y_{0}\right\}}\right) \subset$ $\left\{x_{0}\right\}$. It follows from Lemmas 5 and 7 applied to $T^{-1}$ that $T^{-1} \xi_{\left\{y_{0}\right\}}=\xi_{\left\{x_{0}\right\}}$, and we are done.

LEMMA 9. Suppose that $T \xi_{\left\{x_{0}\right\}}=\xi_{\left\{y_{0}\right\}}$, with $x_{0} \in X$ and $y_{0} \in Y$. If $f \in B V(X)$ satisfies $f\left(x_{0}\right)=0$, then $(T f)\left(y_{0}\right)=0$.

Proof: Suppose that $(T f)\left(y_{0}\right) \neq 0$. Then consider $\alpha \in \mathbb{C}$ such that $|\alpha|=2\|f\|$ and such that there is no $r>0$ satisfying $r \alpha=(T f)\left(y_{0}\right)+\alpha$. It is clear that $C\left(f+\alpha \xi_{\left\{x_{0}\right\}}\right)=$ $\{\alpha\}$, and consequently, by Lemma 3, there exists $r>0$ such that $r \alpha \in C\left(T\left(f+\alpha \xi_{\left\{x_{0}\right\}}\right)\right)$. We obtain a contradiction with the fact that $C\left(T\left(f+\alpha \xi_{\left\{x_{0}\right\}}\right)\right)=\left\{(T f)\left(y_{0}\right)+\alpha\right\}$, and conclude that $(T f)\left(y_{0}\right)=0$.

THEOREM 10. There exists a monotonic bijective map $h: Y \rightarrow X$ such that for every $f \in B V(X)$ and for every $y \in Y$,

$$
(T f)(y)=f(h(y))
$$

Proof: Take $y \in Y$. By Corollary 8 applied to $T^{-1}$, there exists $x \in X$ such that $T \xi_{\{x\}}=\xi_{\{y\}}$. This allows us to define a map $h: Y \rightarrow X$, by $h(y):=x$, where $x$ is obtained from $y$ as above. Also, by Lemma $9,(T f)(y)=0$ whenever $f(h(y))=0$. Now fix $y \in Y$, take $g \in B V(X)$, and let $\alpha:=g(h(y))$. It is clear that $g=\alpha+(g-\alpha)$. Taking into account that $T 1=1$ and $(g-\alpha)(h(y))=0$, we have that $(T g)(y)=g(h(y))$.

On the other hand it is clear that $h$ is injective. It is also surjective, as we can see by applying Corollary 8 . We show finally that $h$ is monotonic. First we take $I \in \mathfrak{I}_{\leftarrow}$, and let $J \in \mathfrak{I}_{\leftarrow} \cup \mathfrak{I}_{\rightarrow}$ such that $T \xi_{X \cap I}=\xi_{Y \cap J}$ (see Lemma 5 ): we claim that if $J$ belongs to $\mathfrak{I}_{\leftarrow}$, then $h$ is increasing, and that if $J$ belongs to $\mathfrak{I}_{\rightarrow}$, then $h$ is decreasing. For, suppose that $J \in \mathfrak{I}_{\leftarrow}$, and that $y_{1}<y_{2}, y_{1}, y_{2} \in Y$. It is clear that we have that either $\left(-\infty, y_{1}\right] \subset J$ or $J \subset\left(-\infty, y_{1}\right]$. In both cases, by Lemmas 5 and 7 applied to $T^{-1}$, we have that $c\left(T^{-1} \xi_{Y \cap\left(-\infty, y_{1}\right]}\right)=X \cap K$, where $K$ belongs to $\mathfrak{I}_{\leftarrow}$. From the process above we 
deduce that $\left(T^{-1} \xi_{Y \cap\left(-\infty, y_{1}\right]}\right)\left(h\left(y_{1}\right)\right)=1$ and $\left(T^{-1} \xi_{Y \cap\left(-\infty, y_{1}\right]}\right)\left(h\left(y_{2}\right)\right)=0$, which clearly implies $h\left(y_{1}\right)<h\left(y_{2}\right)$. Hence $h$ is increasing. The case when $J \in \mathfrak{I}_{\rightarrow}$ yields in a similar way that $h$ is decreasing.

\section{REFERENCES}

[1] J. Araujo and J.J. Font, 'Linear isometries between subspaces of continuous functions', Trans. Amer. Math. Soc. 349 (1997), 413-428.

[2] M. Cambern, 'Isometries of certain Banach algebras', Studia Math. 25 (1965), 217-225.

[3] K. Jarosz and V.D. Pathak, 'Isometries and small bound isomorphisms of function spaces', in Lecture Notes in Pure and Appl. Math. 136 (Dekker, New York, 1992), pp. 241-271.

[4] V ח Pathak, 'Tinear isnmetries of snaces of absolutely continuous functions', Canad. J. Math. 34 (1982), 298-306.

[5] N.V. Rao and A.K. Roy, 'Linear isometries of some function spaces', Pacific J. Math. 38 (1971), 177-192.

Departamento de Matemáticas

Estadística y Computación

Universidad de Cantabria

Facultad de Ciencias

39071 Santander

Spain

e-mail: araujo@matesco.unican.es 